\title{
Long Bone X-ray Image Stitching using C-arm Motion Estimation
}

\author{
Lejing Wang ${ }^{1}$, Joerg Traub ${ }^{1}$, Sandro Michael Heining ${ }^{2}$, Selim Benhimane ${ }^{1}$, \\ Ekkehard Euler ${ }^{2}$, Rainer Graumann ${ }^{3}$, Nassir Navab $^{1}$ \\ ${ }^{1}$ Chair for Computer Aided Medical Procedures (CAMP), TU Munich, Germany \\ ${ }^{2}$ Trauma Surgery Department, Klinikum Innenstadt, LMU Munich, Germany \\ ${ }^{3}$ Siemens SP, Siemens Medical Solutions, Erlangen, Germany \\ wangle@in.tum.de
}

\begin{abstract}
In this paper, we propose a novel method to generate panoramic X-ray images intra-operatively by using the previously introduced camera augmented mobile C-arm by Navab et al. [1]. This advanced mobile $\mathrm{C}$-arm system acquires registered X-ray and optical images by construction, which facilitates the generation of panoramic X-ray images based on the motion estimation of the X-ray source. Visual marker tracking is employed to estimate the camera motion and this estimated motion is also applied to the X-ray source. Our proposed method is suitable and practical for intra-operative usage generating panoramic X-ray images without the requirement of a fronto-parallel setup and overlapping X-ray images. The results show that the panoramic X-ray images generated by our method are accurate enough (errors less than 1\%) for metric measurements and promise suitability for intra-operative clinical applications in trauma surgery.
\end{abstract}

\section{Introduction}

Mobile C-arms are an everyday tool to acquire X-ray images in the operating room during surgery. Currently, long bone fracture fixation heavily relies on intraoperative X-ray images. The limited field of view is a drawback of mobile $\mathrm{C}$-arm imaging. In long bone fracture fixation surgery, surgeons need to measure the length of the bone to be reconstructed and align the bone fragments. Single $\mathrm{X}$-ray image can not visualize the entire long bone. Surgeons are required to acquire several individual X-ray images and correlate them.

Panoramic X-ray images can be obtained by stitching many X-ray images. For obtaining an X-ray panoramic image intra-operatively one method was proposed by Yaniv and Joskowicz [2] using a standard mobile C-arm. This method introduces an orthopaedic radiolucent X-ray ruler placed along and parallel to the bones. It uses the graduations of the ruler in the images to estimate the planar transformation by a feature-based alignment method and requires the user to manually select the reconstruction plane in order to compensate for parallax effects on that plane. However, this method requires overlapping areas between two consecutive X-ray images to estimate the planar transformation and thus 
requires additional radiation. Furthermore, it requires a frontal parallel C-arm setup, i.e. the ruler plane must be parallel to the detector plane of $\mathrm{C}$-arms.

The CamC system that extends a regular mobile C-arm by a video camera [1] was proposed for X-ray and video image overlay. By construction and one time calibration of the device, the X-ray system and the camera have the same projection geometry. Using the video images for estimating the X-ray motion facilitates the creations of panoramic X-ray images. The major advantages over previous proposed solutions are the reduction of ionizing radiation since no overlapping regions of X-ray images are required, not requiring frontal parallel C-arm setup, and enabling metric measurements.

\section{Methods}

During the one time calibration of the CamC system, the X-ray source and the camera optical center are aligned, and then a planar homography is estimated for X-ray and video image overlay. This one time calibration results in that the X-ray source and the video camera have the same intrinsic and extrinsic parameters [1]. Therefore, all the poses and motions estimated using the video camera directly correspond to the X-ray.

The key step of image stitching is the estimation of the planar transformation to align images. The camera pose of acquiring the first image is defined as the world coordinate system, and the second image is obtained after a rotation $R \in \mathbb{R}^{3 \times 3}$ and a translation $t \in \mathbb{R}^{3}$ of the camera. In [2], the planar homography that aligns the first camera image to the second camera image is defined by

$$
H=K R K^{-1}+\frac{1}{d} K t n^{T} K^{-1}
$$

where $K \in \mathbb{R}^{3 \times 3}$ is the intrinsic matrix of the camera. $H$ is valid for all image points whose corresponding space points are on the same plane, called stitching plane, defined by the normal vector $n \in \mathbb{R}^{3}$ and distance $d$ to the origin in the world coordinate system. However, any structure that is not within this stitching plane in 3D space will get ghosting or blurring effects also refered to as parallax.

Thanks to the CamC system, we can make use of the video images in combination of a visual square marker tracking system [3] for C-arm pose estimation. In our implementation, we designed a planar marker pattern, in which all the square markers can be uniquely detected. The corners of the square marker can be extracted with subpixel accuracy and used as feature points. Having the marker pattern with known geometries and uniquely detectable markers, we are able to establish point correspondences between the 2D image points and 3D space points in the marker plane. Based on these point correspondences, the pose of the video camera (the $\mathrm{X}$-ray) with respect to the marker pattern is computed by using OpenCV libraries [4].

To generate panoramic images, the first acquired X-ray image is defined as the reference image, and all others will be registered into coordinate system of this first image. The homography that transforms the $i$-th images to the 
first image coordinate system will be calculated according to equation 1 . The intrinsic parameter $K$ was obtatined during the calibration of the CamC system. Let $R_{i}^{p} \in \mathbb{R}^{3 \times 3}$ and $t_{i}^{p} \in \mathbb{R}^{3}$ be the $\mathrm{X}$-ray source pose in the marker coordinate system for the $i$-th X-ray image. The X-ray source motion, $R_{i}$ and $t_{i}$, from the pose of the $i$-th image to the pose of the first image can be computed by

$$
\left[\begin{array}{cc}
R_{i} & t_{i} \\
0 & 1
\end{array}\right]=\left[\begin{array}{cc}
R_{1}^{p} & t_{1}^{p} \\
0 & 1
\end{array}\right]\left[\begin{array}{cc}
R_{i}^{p} & t_{i}^{p} \\
0 & 1
\end{array}\right]^{-1}
$$

To obtain the homography, the plane parameters are still missing. The parameters of the marker plane in the marker coordinate system are known and represented by $\hat{n}$ and $\hat{d}$. Let $n_{i}$ and $d_{i}$ be the parameters of this marker plane in the camera coordinate system of acquiring the $i$-th image. In [5], we have

$$
\left[\begin{array}{l}
n_{i} \\
d_{i}
\end{array}\right]=\left[\begin{array}{cc}
R_{i}^{p} & t_{i}^{p} \\
0 & 1
\end{array}\right]^{-T}\left[\begin{array}{l}
\hat{n} \\
\hat{d}
\end{array}\right]
$$

So far, we are able to establish a homography, transforming the $i$-th image to the first image coordinate system, which is valid only for image points whose corresponding space points are on the exact marker plane. To obtain the homography valid for the bone plane (i.e. the tibial plane or the nail plane in the intramedullary tibial fracture reduction surgery), which is used for panoramic $\mathrm{X}$-ray images and metric measurements, our method relies on two constraints: 1) the bone plane is placed roughly parallel to the marker plane; 2)The distance between the marker plane and the bone plane is known or estimated. The first constraint provides us the normal of the bone plane and the second constraint gives the distance of this bone plane to the origin in the world coordinate system. With these two constraints, we have the plane equation for the bone plane, and thus the homography valid for the bone plane is calculated and parallax effects on this plane is reduced.

For metric measurements, let $P=[x, y, z]^{T}$ be a $3 \mathrm{D}$ space point on the space plane defined by normal $n$ and distance $d$ in the camera coordinate system, and $m$ be its image projection expressed in homogeneous coordinates. In the camera coordinate system, we have

$$
P=\left(d K^{-1} m\right) /\left(n^{T} K^{-1} m\right)
$$

With the known plane parameters in the camera coordinate system, we can derive the coordinates of space points within this plane from their projections. Therefore, metric measurements on the image are possible for this plane. In our stitching algorithm, all images are registered into the first image coordinate system. Therefore the bone plane parameters in the first camera coordinate system are needed for metric measurements.

\section{Experiments and results}

The phantom (Fig. 1) used throughout all our experiments is constructed to have two parallel planes. On the upper plane, the marker plane for motion 
Table 1. Actual and measured distances/angles between the spherical X-ray markers on the panoramic images in $\mathrm{mm} /$ degrees.

\begin{tabular}{lcclcc}
\hline \multicolumn{2}{l}{$\begin{array}{l}\text { Distances } \\
\text { actual }\end{array}$} & measured & error & Angles & \\
& actual & measured & error \\
\hline 240 & 238.8533 & $1.1467 \pm 0.5423$ & 90 & 90.2657 & $0.2657 \pm 0.0369$ \\
480 & 477.7680 & $2.2320 \pm 0.1556$ & 161.0754 & 161.3224 & $0.2470 \pm 0.0577$ \\
\hline
\end{tabular}

estimation, the marker pattern is attached. On the lower plane, the bone plane, we placed X-ray markers with known distances for metric measurements or a bone phantom for feasibility tests.

The distance is defined by clicking two points on the image (three points for an angle). To determine the accuracy of metric measurements, we used spherical X-ray markers instead of a bone, since we can extract the center of a circle with sub-pixel accuracy. The ground truth of metric measurements is built by attaching X-ray markers on a pattern with known metric properties. The accuracy of the attachments was controlled by a ruler, thus the error in the ground truth was confirmed to be below $1 \mathrm{~mm}$. Our phantom with the X-ray marker was placed on the operating table (Fig. 1(b)) such that the stitching plane deviates several degrees from the image intensifier to simulate the general cases. While acquiring video and $\mathrm{x}$-ray images, the operating table was moved through the $\mathrm{C}$-arm and the panoramic image was created. Then we did the measurements on the generated panoramas. The same experiment was repeated ten times. Table 1 shows the distance and angle measurement results on the panoramic images. In order to investigate the clinical value, we performed preclinical experiments and generated panoramic X-ray images of a bone phantom. Figure 2 shows

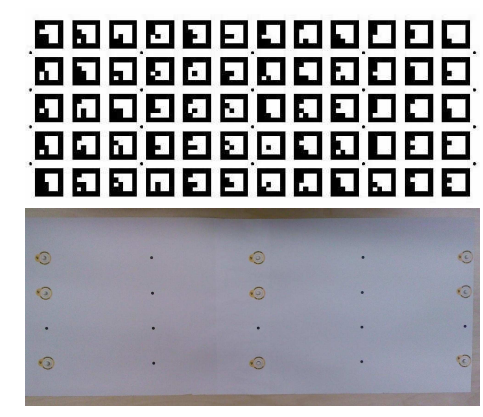

(a) The marker pattern (top) and (b) X-ray markers (bottom).
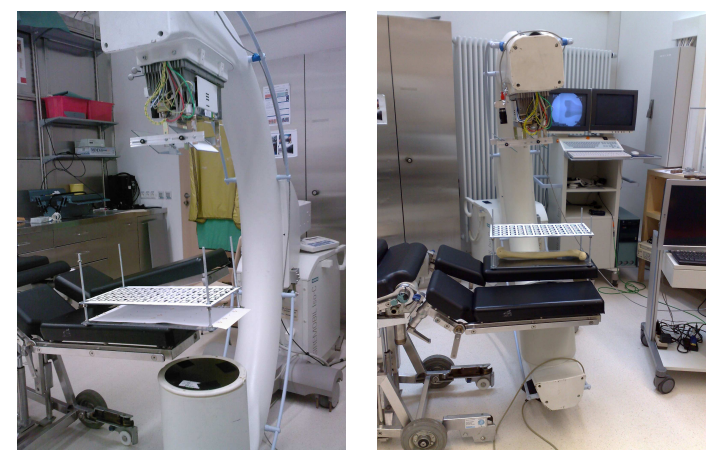

(b) The system setup for

c) The system setup for feasibility tests.

Fig. 1. Our designed phantom for experiments. The marker pattern is attached on the upper plane. The X-ray marker and the bone are placed on the lower plane. 
Fig. 2. The panoramic X-ray image generated from four X-ray images by our method.

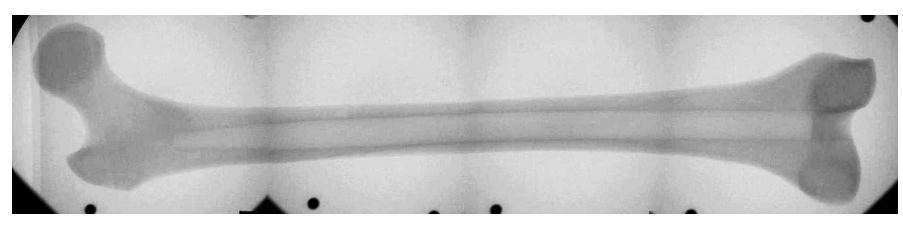

the panoramic X-ray image generated from four individual X-ray images by our method.

\section{Discussion and conclusion}

We presented a new method to generate panoramic radiographs intra-operatively by using the CamC system and a planar maker pattern. The proposed solution integrates smoothly into the surgical procedure as the square marker pattern is only needed during generation of panoramic X-ray images and removed from the complete surgical task. Our method is independent from overlapping X-ray regions and does not require a frontal parallel C-arm setup. However, the accuracy of estimating the transformation valid for the bone plane depends on the estimated distance between the marker plane and the bone reconstruction plane. In general, this distance is not trivial to recover. In our preclinical phantom setup we can directly measure this distance. Appropriate methods have to be incorporated to define the bone plane. Clinical tests in the near future will show the feasibility and the advantage of metric distance and angle measurements within the surgery room based on a standard C-arm. This will allow surgeons to ensure and validate during trauma reduction surgery the quality of their treatment.

\section{References}

1. Navab N, Mitschke M, Bani-Hashemi A. Merging visible and invisible: Two cameraaugmented mobile C-arm (CAMC) applications. In: Proc. IEEE and ACM Int'l Workshop on Augmented Reality. San Francisco, CA, USA; 1999. p. 134-141.

2. Yaniv Z, Joskowicz L. Long bone panoramas from fluoroscopic x-ray images. IEEE Trans Med Imaging. 2004;23(1):26-35.

3. Zhang X, Fronz S, Navab N. Visual marker detection and decoding in AR systems: A comparative study. Proc IEEE ISMAR), October 2002. 2002.

4. OpenCV. http://opencvlibrary.sourceforge.net/wiki-static/.

5. Hartley R, Zisserman A. Multiple View Geometry in Computer Vision. New York, NY, USA: Cambridge University Press; 2003. 